\title{
Editorial
}

\section{Anesthesia and end stage renal failure: is TIVA an advance?}

I $\mathrm{N}$ the 1981 edition of the text "Anesthesia", the general clinical recommendations made for the management of chronic renal failure (CRF) patients are still acceptable today. Bastron suggested that "patients on hemodialysis undergoing elective surgery should be dialyzed the day prior to surgery; that all patients with chronic renal failure should have adequate control of hypertension by anti hypertensive drugs or by ultrafiltration with the dialysis machine; and that the serum potassium be less than $5.5 \mathrm{mEq} \cdot \mathrm{L}^{-1}$ on the day of operation." If this is the case and the clinical principles were well understood in 1981, one has to ask if subsequent research has effectively altered the anesthetic management of patients with end-stage renal disease (ESRD).

The therapeutic tools available to the clinician, as well as our understanding of the pathophysiology have altered the management of anemia, hypertension and systemic coagulation abnormalities associated with CRF. These factors contributed to increased mortality and morbidity of ESRD patients and some have anesthetic implications. ${ }^{2}$ For example, about $90 \%$ of dialyzed patients had anemia resulting from erythropoietin deficiency, and the introduction of recombinant human erythropoietin (epoetin) therapy significantly reduced the need for red cell transfusions and improved exercise tolerance. ${ }^{2}$ The mean hematocrit of patients in ESRD programs is much improved and averaged $31 \%$ in the 1995 Medicare End Stage Renal Disease Program. ${ }^{2}$ The anemia of CRF was associated with left ventricular hypertrophy, an independent risk factor for early death. ${ }^{3}$ Anesthesiologists recognized that CRF patients had a bleeding tendency, however DDAVP, by stimulating endothelial release of VWF-VIII, ameliorates this problem. The management of hypertension and cardiovascular complications was difficult, but with improved intraopera- tive cardiac monitoring, myocardial ischemia is now detectable and treated aggressively. Beta blockade and angiotensin converting enzyme inhibitors minimize perioperative hypertension. In hemodynamically unstable patients, either peritoneal dialysis or continuous veno-venous hemodialysis optimize fluid and electrolyte balance before surgery. Intraoperative hemodialysis is now used, particularly during cardiac surgery, if needed. It is clear that both preoperative and intraoperative care has improved, largely as a result of applying advances of medical management to the perioperative period.

Has recent anesthesia research contributed equally to progress in ESRD patient care? Chronic renal failure still represents an increased operative risk. This risk is not only related to the abnormal clearance of anesthetic agents but, more importantly, is a result of systemic organ dysfunction caused by the underlying disease or renal replacement therapy itself. Modern neuromuscular blocking drugs, opioids and volatile agents, potentially reduce only one element of overall perioperative risk. Altered drug handling in CRF includes decreased drug delivery to the kidney (reduced renal blood flow), increased unbound fraction of highly protein bound agents (hypoalbuminemia and acidosis) and decreased removal of drug from blood.

As new anesthetic agents and techniques were introduced, theoretical advantages were anticipated for CRF patients. The complexity and interindividual variability in drug handling has made titration of inhalational anesthetics a popular, safe technique. In this issue of the journal, Dahaba et al. report that patients with ESRD can be anesthetized with Total Intravenous Anesthesia (TIVA) using remifentanil, propofol and cisatracurium. ${ }^{4}$ There is no doubt that TIVA can be used in these patients but there are no convincing data to show that outcome is improved by 
TIVA as opposed to inhalational anesthesia. Experienced clinicians argue that the ease of titrating inhaled volatile agents to effect, has benefits that are not different from TIVA. Just because we can use these new intravenous agents, should we?

Drugs such as remifentanil, cisatracurium and propofol are reasonable choices in CRF as accumulation is less likely. Altered pharmacokinetic and pharmacodynamic responses to these agents can still occur. Interindividual pharmacokinetic variablility refers to the unique drug disposition of each patient. ${ }^{5}$ Over time, many factors explain differing blood and brain concentrations among individuals. When coupled with pharmacokinetic changes related to age, plasma volume status and duration of drug administration, the physiological variability in CRF is complex. These parients may arrive in the operating room in an intravascularly volume depleted state after recent dialysis. Thus, they are prone to hypotensive responses to vasodilating drugs with negative inotropic effects. Dahaba's study demonstrates that TIVA produced more hypotension in ESRD patients than in control subjects and required more ephedrine, despite lower concentrations of remifentanil. Thus, physiological pharmacokinetic variability remains an issue using TTVA, as it does with all potent anesthetics, including inhalational agents.

Pharmacodynamic variability is the difference in drug action or effect with the same plasma concentration. Some dynamic responses of anesthetic drugs can be measured (eg. muscle twitch height) and related to plasma concentration. There is also interindividual variability in pharmacodynamic responses. ${ }^{5}$ One important class of anesthetic agents that are excreted largely by the kidney are the lipid insoluble, highly ionized neuromuscular blocking drugs. ${ }^{6}$ Decreased renal clearance together with an increased volume of distribution account for delayed recovery from longer acting relaxants (eg. pancuronium and $d$-tubocurarine). With the introduction of intermediate-acting, non-depolarizing relaxants, the effect of ESRD on pharmacodynamic variability has been reduced. The pharmacodynamic and pharmacokinetic behaviour of atracurium and cisatracurium are minimally altered in CRF There are multiple pathways for elimination of atracurium and only about $10 \%$ of the drug is excreted by the kidney in $24 \mathrm{hr}$. The plasma clearance of rocuronium is not affected by renal dysfunction, but patients with renal failure have a larger volume of distribution and the terminal half-life is, therefore, longer. This emphasizes that cis-atracurium is not the only relaxant that can be used safely in ESRD patients, and emphasizes that the margin of safety is improved compared with longer acting agents. Whether using
TIVA or not, careful titration of reduced muscle relaxant dosage is still required.

Before one advocates any specific technique or agent for CRF patients, more than the pharmacodynamics and pharmacokinetics of the drug should be considered. The current method of TIVA administration involves an infusion using a "calculator pump" that allows a continuous infusion. A new drug delivery system will be necessary, if TIVA is to offer the anticipated additional advantages. This system uses an "intelligent pump" that incorporates pharmacokinetic models for these drugs to allow maintenance of constant blood levels by exponentially decreasing infusion rate overtime. Such computer-assisted, continuous infusion (CACI) technology produces less variability in plasma drug levels. To make TIVA preferable to inhalational agents for ESRD patients, a closed loop system to titrate the anesthetic agent to a predetermined anesthetic effect seems essential. The development of the bispectral EEG signal (BIS) and its use with CACI technologies may make TIVA a preferred clinical reality. ${ }^{5}$ At this time, it seems more important how one administers an anesthetic than whether TIVA is used.

There is little doubt that the application of research in the medical management of CRF since 1981 has improved our ability to maintain oxygen delivery and cardiovascular stability in the perioperative period. The development of intermediate-acting relaxants, advances in opiate pharmacology and the use of continuous propofol infusions offer potential advantages to patients with ESRD. Whether TIVA, incorporating these drugs, represents a major advance over the use of intermediate acting relaxants with volatile anesthetics remains unproven. The challenge is to demonstrate that anesthesia research provides improved outcomes that are meaningful to patients. In dealing with ESRD patients, the complex interindividual pharmacokinetic and pharmacodynamic changes require that we use target-controlled infusions, probably combining CACI techniques with BIS. The need for such target controlled infusions will be explored in the next few years. However, clinical judgement in the administration of anesthesia for these complex patients will remain the essential issue. 


\section{L'anesthésie et le stade d'insuffisance rénale ter- minale : l'AEI, un pro- grès?}

Présentées dans l'édition de1981 d' "Anesthesia»", les recommandations cliniques générales sur la ligne de conduite à adopter avec les patients atteints d'insuffisance rénale chronique (IRC) demeurent applicables. Bastron propose que «les patients sous hémodialyse, subissant une intervention élective, soient dialysés le jour précédant l'opération; qu'un contrôle approprié de l'hypertension soit assuré dans tous les cas d'insuffisance rénale chronique par des médicaments ou par l'ultrafiltration au moyen de l'hémodialyseur; que le potassium sérique soit plus bas que $5,5 \mathrm{mEq} \cdot \mathrm{L}^{-1}$ le jour de l'intervention.» Si c'est le cas et si les principes cliniques ont été bien compris en 1981, on doit se demander si les recherches ultérieures ont effectivement modifié la façon de procéder à l'anesthésie des patients au stade d'insuffisance rénale terminale (SIRT).

Les outils thérapeutiques du clinicien, de même que nos connaissances physiopathologiques ont transformé le traitement de l'anémie, de l'hypertension et des anomalies de la coagulation générale associées à l'IRC. Ces complications contribuent à accroître la mortalité et la morbidité des patients au SIRT et certaines ont des implications anesthésiques. ${ }^{2}$ Par exemple, environ $90 \%$ des patients dialysés ont présenté de l'anémie consécutive à une déficience d'érythropoḯtine et l'avènement de la thérapie à l'érythropoiétine humaine recombinante a réduit de façon significative la nécessité de transfusions de globules rouges et a amélioré la tolérance à l'effort. ${ }^{2}$ L'hématocrite moyen des patients participants aux programmes du SIRT a été beaucoup amélioré et se situait, par exemple, à 31 \% dans le Medicare End Stage Renal Disease Program (programme américain). ${ }^{2}$ L'anémie de l'IRC était associée à l'hypertrophie du ventricule gauche, un facteur de risque indépendant de mort prématurée. ${ }^{3}$ On a reconnu qu'avec l'IRC il y a une tendance aux saignements, quoique la DDAVP, en stimulant la libération endothéliale du VIII/VWF, améliore la situation. Le traitement laborieux de l'hypertension et des complications cardiovasculaires s'est amélioré avec le monitorage cardiaque peropératoire, l'ischémie myocardique étant maintenant détectable et traitée énergiquement. Par ailleurs, le bêtablocage et l'inhibi- tion de l'enzyme de conversion de l'angiotensine réduisent l'hypertension périopératoire. Chez les patients dont l'hémodynamie est instable, la dialyse péritonéale ou l'hémodialyse veino-veineuse continue optimalise l'équilibre hydro-électrolytique. L'hémodialyse peropératoirc est utilisée au besoin actuellement, surtout pendant la chirurgie cardiaque. Les soins préopératoires et peropératoires se sont donc améliorés et c'est beaucoup grâce à la mise en application des perfectionnements de la gestion médicale périopératoire.

Les recherches récentes en anesthésie ont-elles contribué également à l'évolution des soins au SIRT? L'insuffisance rénale chronique représente toujours un grand risque opératoire qui n'est pas seulement relié à la clairance anormale des agents anesthésiques. Il est davantage le résultat d'un dysfonctionnement organique général causé par la maladie sous-jacente ou la thérapie de suppléance rénale elle-même. Les myorelaxant, les opioïdes et les anesthésiques volatils modernes pourraient ne réduire qu'un élément du risque périopératoire global. Modifier le maniement des médicaments dans les cas d'IRC signifie un apport réduit de médicament aux reins (réduction du débit sanguin rénal), une augmentation de la fraction libre des agents fortement liés aux protéines (hypoalbuninémie et acidose) et une élimination réduite du médicament contenu dans le sang.

Au fur et à mesure qu'on introduisait de nouveaux anesthésiques et techniques, on s'attendait, en théorie, à des avantages pour les patients atteints d'IRC. Étant donné la complexité et la variabilité interindividuelle de la réponse aux médicaments, le titrage des anesthésiques par inhalation est devenu une technique populaire et sûre. Dans le présent numéro, Dahaba et coll. affirment que les patients en SIRT peuvent recevoir une anesthésie exclusivement intraveineuse (AEI) de rémifentanil, de propofol et de cisatracurium. ${ }^{4}$ L'AEI peut sans contredit être utilisée au SIRT, mais rien ne prouve que le résultat soit meilleur qu'avec l'anesthésie par inhalation. Des cliniciens expérimentés soutiennent que la facilité du dosage des anesthésiques volatils en fonction de l'effet offre des avantages comparables à ceux de l'AEI. Par conséquent, la seule possibilité d'utiliser ces nouveaux agents intraveineux justifie-t-elle leur emploi?

Des médicaments comme le rémifentanil, le cisatracurium et le propofol constituent de bons choix, leur accumulation étant probablement plus faible. Mais il est possible que le comportement pharmacocinétique et pharmacodynamique se modifie : c'est la variabilité pharmacocinétique interindividuelle qui représente, pour chaque patient, une façon unique de réagir au médicament. ${ }^{5}$ Avec le temps, de nombreux 
facteurs permettent d'expliquer les différences interindividuelles de concentrations sanguines et cérébrales. Si on les combine aux changements pharmacocinétiques reliés à l'âge, à l'état du volume plasmatique et à la durée de l'administration du médicament, la variabilité physiologique de l'IRC devient complexe. Les patients peuvent se présenter en salle d'opération avec une déplétion du volume intravasculaire à la suite d'une récente dialyse s'exposant alors à l'hypotension liée aux médicaments vosodilatateurs et à des effets inotropes négatifs. L'étude de Dahaba démontre que l'AEI a produit plus d'hypotension chez les patients en SIRT que chez les sujets témoins et a nécessité plus d'éphédrine en dépit de plus faibles concentrations de rémifentanil. Ainsi, la variabilité pharmacocinétique compte toujours avec l'AEI, comme c'est le cas avec tous les anesthésiques puissants, y compris les agents par inhalation.

La variabilité pharmacodynamique concerne la différence d'action, ou d'effet, du médicament pour une même concentration plasmatique. On peut mesurer certaines réponses dynamiques aux anesthésiques (par ex., l'importance de la contraction musculaire) et liées à la concentration plasmatique. Il y a aussi une variabilité interindividuelle des réponses pharmacodynamiques. ${ }^{5}$ Une classe importante d'anesthésiques fortement éliminés par le rein est représentée par les myorelaxants qui sont lipo-insolubles et hautement ionisés. ${ }^{6} \mathrm{La}$ baisse de la clairance rénale et l'accroissement du volume de distribution expliquent la récupération tardive qui suit l'utilisation des myorelaxants à action prolongée comme le pancuronium et la $d$-tubocurarine). L'introduction des myorelaxants non dépolarisants à action intermédiaire a permis de réduire l'effet du SIRT sur la variabilité pharmacodynamique. Le comportement pharmacodynamique et pharmacocinétique de l'atracurium et du cisatracurium est très peu modifié dans les cas d'IRC. Il y a de multiples voies d'élimination de l'atracurium et seulement $10 \%$ du médicament environ sont éliminés par le rein en $24 \mathrm{~h}$. La clairance plasmatique du rocuronium n'est pas changée par le dysfonctionnement rénal, mais on note, dans ce cas, un plus grand volume de distribution entraînant une demivie d'élimination terminale plus longue. Cela confirme que le cisatracurium n'est pas le seul myorelaxant utilisable de façon sécuritaire chez les patients au SIRT et que sa marge de sécurité est meilleure que celle des anesthésiques à action prolongée. Qu'on utilise l'AEI ou non, un titrage méticuleux d'une baisse du dosage de myorelaxant est toujours nécessaire.

La pharmacodynamie et la pharmacocinétique ne sont pas les seuls paramètres à considérer avant de conseiller quelque technique ou agent spécifique que ce soit pour les patients atteints d'IRC. La méthode habituelle d'administration de l'AEI comporte une perfusion à l'aide d'une «pompe calculatrice» qui permet une perfusion continue. Pour que l'AEI puisse offrir les avantages supplémentaires prévus, il faut un nouveau système de livraison du médicament qui utilise une «pompe intelligente» incorporant les modèles pharmacocinétiques du médicament afin de maintenir des niveaux sanguins constants par une baisse exponentielle de la vitesse de perfusion en fonction du temps. Ces perfusions continues assistées par ordinateur (PCAO) provoquent moins de variations des niveaux plasmatiques de médicament. Un système en boucle fermée apparaît essentiel pour titrer l'anesthésique en fonction d'un effet prédéterminé et pour assurer la supériorité de l'AEI sur l'anesthésie par inhalation au SIRT. Le développement de l'EEG bispectral (BIS) et son utilisation avec les PCAO peuvent faire de l'AEI l'option clinique préférée. ${ }^{5}$ Actuellement, on attache plus d'importance à la façon d'administrer un anesthésique qu'au fait de savoir si l'AEI est utilisée.

Il ne fait pas de doute que, depuis 1981, l'application des recherches au traitement médical de l'IRC a amélioré notre habileté à maintenir la livraison d'oxygène et la stabilité cardiovasculaire périopératoires. La production de myorelaxants à action intermédiaire, les progrès de la pharmacologie des opiacés et l'utilisation de perfusions continues de propofol offrent des avantages potentiels aux patients qui sont au SIRT. Que l'AEI, et ces médicaments, représentent une évolution importante dans l'usage de myorelaxants à action intermédiaire, comparés aux anesthésiques volatils, cela reste à prouver. Le défi est de démontrer que les recherches en anesthésie permettent de meilleurs résultats, significatifs pour les patients. En intervenant au SIRT, les changements pharmacocinétiques et pharmacodynamiques interindividuels complexes nécessitent l'utilisation de perfusions cibles contrôlées, combinant ainsi les techniques de PCAO et le BIS. Les besoins de semblables perfusions cibles contrôlées feront l'objet d'études dans les prochaines années. Cependant, l'enjeu essentiel concerne toujours le jugement clinique qui s'applique à l'administration de l'anesthésie aux patients atteints de maladies complexes.

\section{References}

1 Bastron RD. Anesthesia for genitourinary surgery. In: Miller RD (Ed.). Anesthesia. New York: Churchill Livingstone Inc., 1981: 1061-78.

2 Adamson JW, Eschbach JW. Erythropoeitin for endstage renal disease (Editorial). N Engl J Med 1998; 339: $625-7$. 
3 Foley RN, Parfrey PS, Hartnett JD, Kent GM, Murray $D C$, Barre PE. The impact of anemia on cardiomyopathy, morbidity and mortality in end-stage renal disease. Am J Kidney Dis 1996; 28: 53-61.

4 Dababa AA, von Klobucar F, Rebak PH, List WF. Total intravenous anaesthesia with remifentanyl, propofol and cis-atracurium in end stage renal failure patients. Can J Anesth 1999; 46: 696-700.

5 Reves JG. New concepts in delivering intravenous anesthetic agents. ASA Refresher Course Lectures 1998: 173.

6 Hunter JM. Drug therapy: neuromuscular blocking drugs. N Engl J Med 1995; 332: 1691-9. 